\title{
Recoil effects in neutron activation analysis and radioisotope production: Formulation and practical consequences
}

\author{
H. A. Das
}

ECN P.O. Box 1, 1755 ZG, The Netherlands

(Received August 18, 2006)

In activation analysis, recoil causes both contamination from and loss to the packing material. In isotope production, recoil may be used to obtain carrier-free radionuclides. Both phenomena depend on irradiation geometry and recoil range. Their formulation and that of experimental range determinations is addressed for common irradiation practice.

\section{Introduction}

Recoil effects, usually referred to as SzilardChalmers processes, are described in many textbooks, e.g., in References 1-3. They are caused by the emission of an $\alpha, \beta$ or $\gamma$ during activation or decay. The reacting nucleus acquires a high kinetic energy and charge which it looses by interaction with the surrounding medium. This "hot atom" chemistry is summarized in, e.g., References 4 and 5.

In activation analysis and isotope production with neutrons, recoil is caused by prompt gamma-emission, causing transport of activated nuclei across phase boundaries. Its effect for the most common irradiation geometries is considered here. Pertinent formulation permits corrections and may serve as a basis for the determination of recoil ranges.

\section{Formulation $^{6}$}

The escape probability $K$ for a given range $L$, of the recoil nuclei, generated at random in a homogeneous matrix, has to be defined for common sample shapes.

The probability for emission from depth $x, k_{x}$, is integrated over the range $L$, to yield the average, $K$, which then is transformed in the fraction $F$, emitted from the sample as a whole. Obviously, $F$ tends to zero with increasing sample dimensions.

Expressions for straight, concave and convex and borderlines in a plane and for the corresponding threedimensional configurations of spherical shell, sphere and cylinder are derived in Reference 6.

Cases of paramount interest in neutron activation analysis and isotope production are:

Flat specimen of thickness D:

$$
F=0.25 \cdot L / D
$$

Spherical shell or sphere of radius $R$ :

$$
\begin{gathered}
F=[0.25\{1-0.4 L / R\}] \cdot\left[\{L / D\} \cdot\left\{1+L / R+1 / 3(L / R)^{2}\right\} /\right. \\
\left./\left\{1+D / R+1 / 3(D / R)^{2}\right\}\right]
\end{gathered}
$$

Cylinder of radius $R$ and length $H$ with $R / H<0.05$ :

Figure 1 gives the ratio $F_{\text {cylinder }} / F_{\text {plane }}$ as a function of $L / R$.

\section{Range of recoil nuclei}

The kinetic energy $E_{r}$, in $\mathrm{keV}$, of a recoil nucleus with mass number $A$ depends on the averaged and weighted square of the prompt gamma-rays, $\left(E_{\gamma}\right)^{2}$ in $(\mathrm{MeV}),{ }^{2}$ as $E_{r}=\left(E_{\gamma}\right)^{2} / 1862 \mathrm{~A}$. Estimates of $\left(E_{\gamma}\right)^{2}$ can be obtained from the tabulation of prompt gamma-energies in Reference 7. The initial velocity $v_{\mathrm{o}}$ in $\mathrm{cm} \cdot \mathrm{s}^{-1}$ of the recoil nucleus follows from $E_{r}$ as $4.4 \cdot 10^{7}\left(E_{r} / A\right)^{1 / 2}$. With $\left(E_{\gamma}\right)^{2}$-values ranging from $\sim 7$ to $\sim 30(\mathrm{MeV})^{2}$ and $A$ from 20 to 200 , initial velocities very widely, from $\sim 0.04 \cdot 10^{7}$ to $\sim 0.5 \cdot 10^{7} \mathrm{~cm} \cdot \mathrm{s}^{-1}$.

As the range of different particles with the same initial energy but different mass $A$ and charge $z$ is about proportional to $A / z^{2}$, an estimate of the range of recoil nuclei in dry air at $15{ }^{\circ} \mathrm{C}$ and atmospheric pressure can be obtained by comparison to the data for $\alpha$-particles as tabulated in Reference 8. Due to the uncertainty in the charge of recoil nuclei and its variation over the range, lower and upper estimates differ by a factor of about five.

Transformation to other matrices by way of the Bragg-Kleeman rule results in the range-estimates of $0.5-2.5 \mu \mathrm{m}$ for polythene, perspex and nylon, $0.7-$ $3.5 \mu \mathrm{m}$ for dry plant material and $0.4-2.0 \mu \mathrm{m}$ for quartz.

Experimental determination is performed, either by collecting in a catcher foil ${ }^{9}$ on the surface or by depth profiling. ${ }^{10,11}$ Preferably, a flat thin specimen is used. With no flux-depression present and $r=$ (combined activity of the two foils covering the flat sides)/(activity of the sample of thickness $D, \mu \mathrm{m})$, one has $L=2 D[r /(1+r)] \mu \mathrm{m}$. 
With a one-side surface area of $10 \mathrm{~cm}^{2}$ and commonly available neutron fluxes, collected activities will range from $\sim 10^{5}$ to $\sim 10^{8} \mathrm{~Bq}$ for $L=1 \mu \mathrm{m}$ and can be determined with good precision.

Depth-profiling of small, homogeneous and perfectly flat samples is possible with a precision polishing apparatus. ${ }^{10}$ Relative error increases with decreasing thickness of the removed layer to reach $\sim 10 \%$ below $0.5 \mu \mathrm{m}$.

For $n$ consecutive abrasions of depth $x_{0}, \mu \mathrm{m}$, each applied to a surface area of $S, \mathrm{~cm}^{2}$, at a concentration of $c_{0}$ nuclei $\cdot \mathrm{cm}^{-3}$, the number of recoil nuclei $N$, collected after the $n$th step is given by:

$N=10^{-6}\left[c_{\mathrm{o}} \mathrm{S}\right] \cdot\left[n x_{0} / 2\right] \cdot\left[1+\left(n x_{0} / L\right)\right]$, provided $n x_{0}<L$.

With $L \sim 10 \mu \mathrm{m}, x_{0} \sim 1-2 \mu \mathrm{m}$ and $\Delta x_{0} / x_{0} \sim 0.05-0.10$, the relative uncertainty in $N$ due to that in $x_{0}$ is of the order of a few percent.

\section{Recoil effects in neutron activation analysis}

The relative change in the number of activated nuclei in the sample, $(\Delta c / c)_{1}$, is obviously given by:

$$
[S / V] \cdot 10^{-4} \cdot\left[\left\{(K L c)_{2}-(K L c)_{1}\right\} / c_{1}\right]
$$

where the indices " 1 " and " 2 " refer to sample and container, respectively, $K$ is the average escape probability over the range $L, S$ is the contact area in $\mathrm{cm}^{2}$ and $V$ is the sample volume in $\mathrm{cm}^{3}$, with again concentrations $c$ in nuclei $\cdot \mathrm{cm}^{-3}$ and ranges $L$ in $\mu \mathrm{m}$.

Using the Bragg-Kleeman rule again, one can rewrite $(\Delta c / c)_{1}$ as:

$$
\left[S K_{1} L_{1} / V\right] \cdot 10^{-4} \cdot\left[\left\{\left(K A^{1 / 2} c\right)_{2} /\left(K A^{1 / 2} c\right)_{1}\right\}-1\right]
$$

Dry plant material in high purity quartz or glass tubes of $2 \mathrm{~mm}$ radius may elucidate the use of this last expression.

With $L \sim 2 \mu \mathrm{m}$ for all materials, irradiation in quartz causes a loss of $\sim 0.05 \%$. Glass implies ratios $c_{2} / c_{1}$ of $\sim 10^{2}$ to $\sim 10^{3}$ and resulting positive biases range from $\sim 10$ to $\sim 100 \%$.

One gram sample divided into 100 equal spheres and suspended in an analyte-free medium will suffer a loss of $0.3 \underline{5} \%$.

Experimental testing of package materials is performed by irradiating ultra-pure active carbon. ${ }^{12}$

\section{Carrier-free radionuclide production by recoil}

The fraction $F$, emitted by a flat specimen of oneside surface area $S\left(\mathrm{~cm}^{2}\right)$ and thickness $D(\mathrm{~cm})$ is 0.50 $L / D$. For thin layers of pure metals with $L \sim 1 \mu \mathrm{m}$ and specific activities of $10^{8}$ to $10^{12} \mathrm{~Bq} \cdot \mathrm{cm}^{-3}$, this results in $10^{4}$ to $10^{8} \mathrm{~Bq} \cdot \mathrm{cm}^{-2}$

The fraction $F_{y}$ deposited at depth $y$ in the receiving phase depends on the range $L_{1}$ in the emitting phase and the ratio $f$ of the ranges in receiver and emitter as:

$$
F_{y}=1 / 4\left[\pi / 2-y / f L_{1}\left\{1-\left(y / f L_{1}\right)^{2}\right\}-\operatorname{arc}\left\{\sin y / f L_{1}\right\}\right]
$$

Deposition probability as a function of $y / f L_{1}=y / L_{2}$ follows as:

$$
R_{y}=\int_{0}^{y} F_{y} \mathrm{~d}\left(y / f L_{1}\right) / \int_{0}^{1} F_{y} \mathrm{~d}\left(y / f L_{1}\right)
$$

and is shown in Fig. 2.

About $80 \%$ of the received activity is deposited at $y / f L_{1}<0.5$. The collection of carrier-free ${ }^{18} \mathrm{~F}$, $\left(T_{1 / 2}=110 \mathrm{~min}\right)$ from teflon on top of a polythene foil is an example. 
H. A. DAS: RECOIL EFFECTS IN NEUTRON ACTIVATION ANALYSIS AND RADIOISOTOPE PRODUCTION

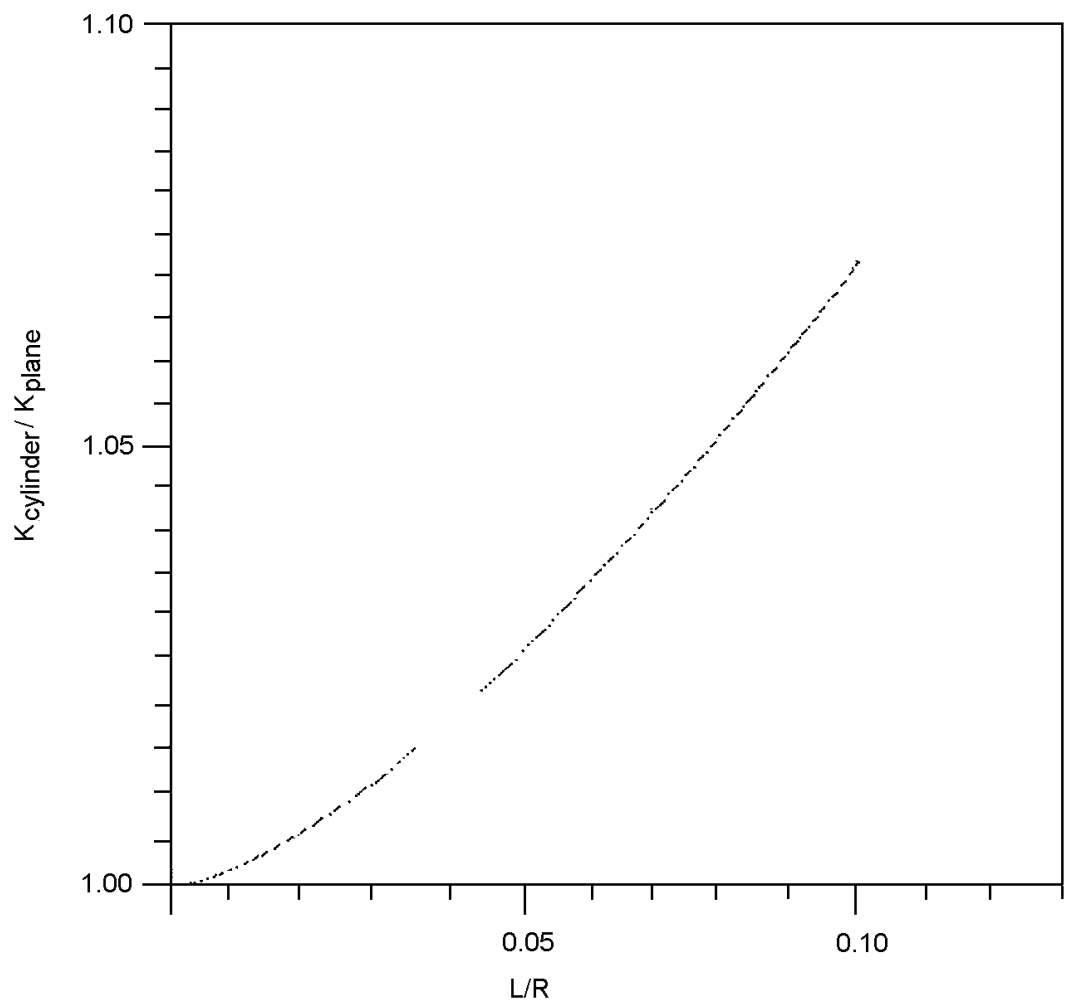

Fig. 1. Ratio $K_{\text {cylinder }} / K_{\text {plane }}$ as a function of $L / R$ for a long cylinder

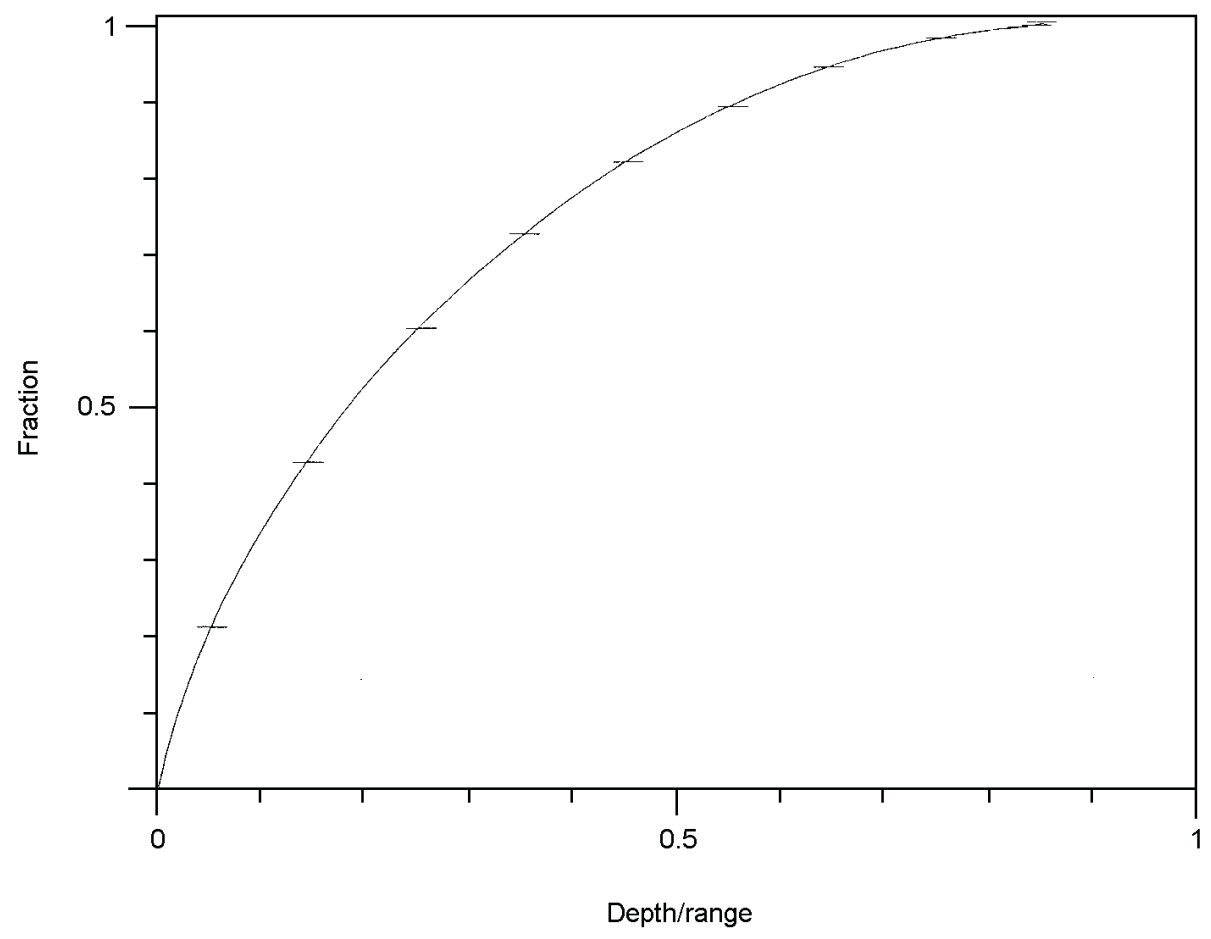

Fig. 2. Cumulative distribution of recoil nuclei as a function of the range in the receiving phase 


\section{Conclusions}

Neutron irradiation implies transfer across phase borders of recoiling radionuclides and thus to losses or contamination, depending on parent nuclides, package material and geometry. These transitions can be formulated in terms of experimentally obtained recoil ranges, obtained by using catcher foils or depthprofiling on the micrometer scale.

In neutron activation analysis the most important effect of recoil is the contamination by the package material. Losses from the sample are usually small.

Collection of recoiled nuclei may be applied for the production of small amounts of carrier-free activities, up to $\sim 10^{8} \mathrm{~Bq}$.

\section{References}

1. M. HAÏSSINSKY, Nuclear Chemistry and its Applications. Addison-Wesley Publishing Co., Inc., Reading, 1964, Chapter 17.

2. G. Friedlander, J. W. Kennedy, J. M. Miller, Nuclear and Radiochemistry, 2nd ed., John Wiley \& Sons, New York, 1964, p. $209 \mathrm{ff}$.

3. H. M. MCKAY, Principles of Radiochemistry, Butterworths, London, 1971, Chapter 8.

4. G. Stöcklin, J. Vernois / R. MuXart (Trs.), Chimie des Atomes Chaudes, Masson et Cie, Paris, 1972.

5. T. Tominaga, E. TaChiKaWA, Modern Hot Atom Chemistry and its Applications, Springer Verlag, Berlin, 1981.

6. Recoil Effects in Neutron Activation Analysis and Radioisotope Production: Formulation and Practical Consequences, Report ECN, February 2005, Available upon request.

7. R. STEPHENSON, Introduction to Nuclear Engineering, McGraw-Hill Book Co., New York, 1958, p. 230.

8. R. D. Evans, The Atomic Nucleus, McGraw-Hill, Inc., New York, 1955, p. 649.

9. K. StrijCKmans, in: Chemical Analysis by Nuclear Methods, Z. B. Alfassi (Ed.), J. Wiley \& Sons, New York, 1994, p. 237.

10. L. M. J. Leblans, M. L. Verheyke, Philips Techn. J., 25 (1963) 103.

11. H. A. DAS, F. H. M. SPIT, J. Radioanal. Nucl. Chem., 117 (1987) 171.

12. The Preparation of Ultrapure Active Carbon, Report ECN, June 1978. 